Acta Crystallographica Section A

Foundations of Crystallography

ISSN 0108-7673

Received 23 March 2004

Accepted 15 July 2004

(C) 2004 International Union of Crystallography Printed in Great Britain - all rights reserved

\section{'In-situ' charge-density studies of photoinduced phenomena: possibilities for the future?}

\author{
Jacqueline M. Cole
}

Department of Chemistry, University of Cambridge, Lensfield Road, Cambridge CB2 1EW, England. Correspondence e-mail: jmc61@cam.ac.uk

Over the past decade, there has been much development in the structure determination of photoinduced species by single-crystal X-ray diffraction. Such work is key to understanding many photoactivated chemical processes and physical properties that are behind phenomena such as photoisomerism, photoinduced chemical reactions, light-induced spin-crossover transitions and molecular excited states that are responsible for many types of fluorescence and phosphorescence. A brief overview of these experimental developments is presented in relation to the attraction of conducting charge-density studies on photoinduced structures. The technical issues regarding possible charge-density studies using these developments, both in the metastable and time-resolved domain, are highlighted in the form of a perspective towards future possibilities for photoinduced charge-density studies. The paper concludes with a summary of further experimental developments that are unfolding and how these may contribute to the ultimate viability of 'in-situ' charge-density studies on photoinduced phenomena.

\section{Introduction}

Photoinduced species occur in a myriad of compounds, ranging from those evident in nature, e.g. chlorophyll, through to technologically important materials such as photodarkening semiconductors and light-emitting-diodes. The photolysis process excites electrons into otherwise inaccessible states, thus inducing a redistribution of electrons within a molecule. In turn, this can lead to atomic displacement within the structure of the material or, more severely, isomerism or solidstate chemical reactions. A wide variety of photophysical properties unfold from these structural perturbations as these cause, for example, associated changes in dipole moments, luminescence or radical formation.

Whilst photophysical properties can be fairly readily measured, their structural origins generally remain elusive except via indirect structural information that has been made available mainly from optical and infra-red spectroscopy. Furthermore, the majority of this indirect structural information emanates from solution-based spectroscopy measurements, and yet, the physical property so often sought is aimed at solid-state devices. The resulting dearth of same-phase comparisons between structure and property hamper implicitly our understanding of the role of structure in a given physical phenomenon.

Crystallography is the ultimate technique for determining the bond-geometry of a molecule with high accuracy and in a three-dimensional representation. Given that such structural characterization is also undertaken using solid-state samples, crystallography would therefore seem to be the ideal tech- nique with which to capture 'in-situ' three-dimensional molecular images of photoinduced states, such that one can understand photophysical processes.

The typically very transient nature of photoinduced processes makes this area of crystallographic development particularly challenging, since the structure determination of photoinduced species that are not very long-lived or metastable (the majority) requires one to embrace time-resolved crystallography. Typically, this time-resolution will be realized by (optical)pump-(X-ray)-probe technology whereby crystallographic measurements are acquired in a stroboscopic fashion, in between the continually repeating pulses of optical excitation over the duration for which the subject molecule remains excited. There are also various practical complications surrounding the optical excitation that must be taken into consideration that are competing with optimal crystallographic experimental conditions (see §2).

Furthermore, high atomic resolution is usually a prerequisite; in the majority of cases, the redistribution of electrons, caused by photoinduction, manifests itself in an associated change in bond distance between two or more atoms within a molecule. For example, a luminescent organometallic material will typically be photoexcited into a state that will effect metal-ligand charge transfer (MLCT). The primary structural perturbation in such a case will be a change in bond length between the metal and ligand(s) concerned. Similarly, an oxidation change may be brought about by photoinduction in a material and in such a case the coordination sphere about the metal ion will contract or expand according to the increase or decrease in oxidation number, respectively. Bond-length 
changes of $0.1-0.2 \AA$ would be typical, but this could be much smaller, particularly in organic compounds; $\mathrm{C}-\mathrm{C}$ bond lengths are shorter than metal-containing bonds and so any changes are inherently harder to detect. Obtaining a sufficient level of atomic resolution to observe the structural perturbation sought thus presents a major experimental challenge in this area.

Ultimately, one would in fact wish to be able to collect data with high enough resolution to perform three-dimensional electron-density distribution mapping via a full multipole charge-density refinement, since a topological representation of the electron density in a molecule is by far the best way to observe the nature of electronic charge-transfer processes that govern these structural perturbations. Furthermore, a value for the solid-state molecular dipole moment, $\mu$, can be afforded from charge-density studies, and its variation relative to the initial ground state could prove very useful, as could a topological analysis of the electrostatic interactions ensuing within the molecule, again available via charge-density studies. However, owing to the large number of parameters required to adequately define multipoles for a charge-density study, together with the restriction that the overall data:parameter ratio should remain sufficient (typically 10:1) for reliable refinement to be carried out, one is limited to using the independent atom model (IAM) in crystallographic structure determination unless suitably careful and extensive data collections can be undertaken. The IAM reveals the bond geometry in a compound by evaluating the distances between the centres of spheres of electron density that are modelled between two given atoms, i.e. there is no explicit regard given to possible electronic polarization effects between bonds, which play a dominant role in photoinduced phenomena.

There is much curiosity as to whether or not charge-density studies on photoinduced species will be possible in the future, and it is the object of this paper to provide a perspective to this end, addressing the various issues that need to be contended with if such experiments are to become viable.

\section{A technique under development: experimental considerations}

Photoactivated processes fall into two categories, the choice of class depending on the extent of structural changes engendered by photoexcitation. Those effects that alter substantially the structure of a compound, for example a photoisomerization process, form one of these classes. The resulting structures are likely to be long-lived or metastable, since a large structural perturbation generally takes a while to revert to its initial non-photoinduced structure, if indeed it can ever overcome the energy activation barrier to return to its initial state.

The structure of a long-lived or metastable photoisomerized state is revealed via three experimental steps: firstly, one determines the ground-state structure of the material using conventional small-molecule single-crystal X-ray diffraction methods; next, the crystal, still mounted on the X-ray diffractometer, is optically pumped for several hours using a suitable light source, this typically being a flash-lamp (broad- band wavelength of light) or a laser (monochromatic source); the light source is then removed and the resulting structure is determined, again using conventional $\mathrm{X}$-ray diffraction methods and under the same experimental conditions as before. The resulting structure from this second data collection will comprise contributions from both the original ground state and the light-induced structure; the light-induced structure cannot generally be obtained experimentally in isolation since, in most photoinduction processes, one cannot achieve $100 \%$ photoconversion in a single crystal. In fact, one generally aims to obtain no more than $20-30 \%$ photoconversion since, beyond this, the integrity of the crystal is endangered; if structural perturbations within a crystal lattice are too great or numerous, the crystal will fracture or explode.

In effect, therefore, structural photoisomerism manifests itself in the same way that static disorder does in a crystal structure; the 'photoisomerized' (minor) component is located in the Fourier map of the second set of data collected, and is modelled and refined as such with an occupancy factor of $1-X$, where $X$ is the occupancy factor of the dominant (major) non-photoactivated structure, initially modelled using the first data set. For example, $\eta^{1}$ to $\eta^{2}$ photoisomerization of the $\mathrm{SO}_{2}$ ligand in the compound, $\mathrm{Ru}\left(\mathrm{SO}_{2}\right)\left(\mathrm{NH}_{3}\right)_{4} \mathrm{Cl} \cdot \mathrm{Cl}$, at a laser wavelength $\lambda=355 \mathrm{~nm}$ yields $\sim 7 \%$ photoisomerization, such that a Fourier difference map of the (light + ground state) - (ground state) model, normalized to the light-induced unit-cell parameters, shows the $\eta^{2}-\mathrm{SO}_{2}$ ligand thus formed (see Fig. 1). The Fourier difference map is thence used to locate the positions of the atoms in this $\eta^{2}-\mathrm{SO}_{2}$ ligand and to refine them as a minor component of the $\mathrm{SO}_{2}$ contribution to the overall crystal structure. Further details of this 'photodifference' type of analysis have been described by Carducci et al. (1997).

More transient photoinduced phenomena, where the lifetime of the photoinduced state, $\tau_{\mathrm{o}}$, is typically $\mathrm{ps}<\tau_{\mathrm{o}}<\mu$ s, fall

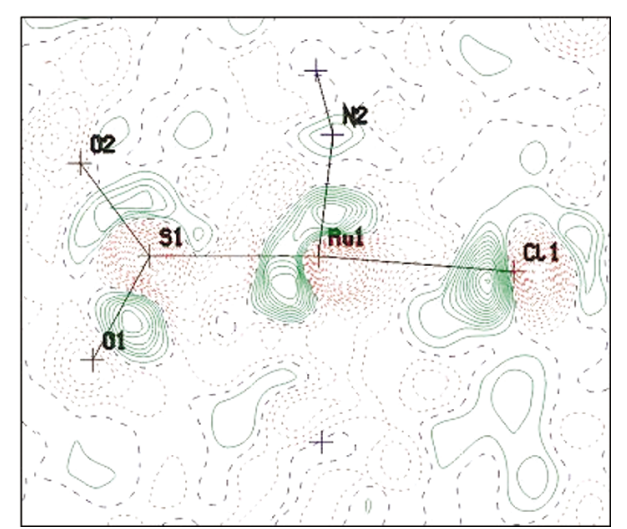

Figure 1

A 'photodifference' map showing the ground state (already modelled) depicted by the black lines together with the electron density associated with the light-induced $\left[\mathrm{Ru}\left(\mathrm{SO}_{2}\right)\left(\mathrm{NH}_{2}\right)_{4} \mathrm{Cl}\right] \mathrm{Cl}$ complex (Kovalevsky et al., $2002,2003)$. In this example, $\mathrm{SO}_{2}$ is the photoactive ligand, undergoing a $\eta^{1}-\mathrm{SO}_{2}$ (end-on) to $\eta^{2}-\mathrm{SO}_{2}$ (side-bound) photoisomerization. The $\mathrm{S}$ atom and one of the $\mathrm{O}$ atoms of the $\eta^{2}-\mathrm{SO}_{2}$-bound ligand are evident in this Fourier difference map by the green peak and more diffuse green area, respectively, on the left of the figure (the other oxygen cannot be seen here as it lies out of the plane). 
into a second category. Here, photostructural changes are very subtle, manifesting themselves, for example, by a change in one of the bond lengths of the molecule (typically $0.1 \AA$ or less), as noted earlier. Again, the level of photoexcitation is unlikely to exceed $10-20 \%$ and, owing to their ephemeral nature, such photostructural effects must be determined using time-resolved stroboscopic pump-probe single-crystal X-ray diffraction techniques.

In order to affect a time structure for such stroboscopic pump-probe experiments, a train of X-ray pulses is created and synchronized to the timing of a laser pulse. The time length of each X-ray pulse must be shorter than the lifetime of the light-induced phenomenon but substantially longer than the laser pulse, such that there is ample time to acquire X-ray diffraction data after the laser has excited the sample but before the light-induced effect has had time to decay appreciatively.

For materials where the photoinduced lifetime is greater than microseconds, a mechanical chopper can be used to generate suitable X-ray pulses. An example of such a device has been described by Ozawa et al. (1998). Where the photoinduced lifetime is at the nanosecond or picosecond level, the temporal structure of the electron beam in the accelerator of a synchrotron can be exploited to obtain nanoto picosecond X-ray pulses. An example of such an experimental setup is the ID9 beamline at the ESRF, Grenoble, France (Wulff et al., 1997), which can extract picosecond X-ray pulses selectively, most commonly from the 16-bunch or single-bunch modes of the ESRF synchrotron beam, with the help of a specially designed chopper on the ID9 instrument that allows the incoming 50-100 ps pulses to reach the diffractometer at a repetition rate that corresponds to that of the laser being used for optical pumping.

The fact that most lasers operate with a typical pulse repetition rate of $10 \mathrm{~Hz}$ (flash-lamp based) or $10 \mathrm{kHz}$ (diode based) means that there is an enormous percentage of deadtime to behold compared with the time of data acquisition, which takes place only when the molecule is in the photoinduced state, i.e. the method affords a very low experimental duty cycle. The data-collection time required for a basic singlecrystal X-ray diffraction structure determination with such pulsing is therefore considerable, bordering on the inexorable in certain cases if other factors cannot be altered to reduce data-collection time.

Data-collection time is commonly reduced by increasing the intensity of diffraction, either by using a stronger X-ray source or by employing a larger crystal for the experiment. However, the optical penetration depth of a crystal is usually of the order of micrometres and so there is an inherent maximum crystal size that can be used. The use of a crystal beyond this maximum size threshold will result in the crystal exploding, since the optical pumping light cannot wholly pass through a sample and so will impart a build-up of local heating effects within the crystal that will lead, in turn, to crystal fracture (see Fig. 2).

Aside from optical penetration depth considerations, a laser often causes crystal decay over time for other reasons. There is therefore also a large incentive, from this perspective, to keep the data-collection times as short as possible.

If the subject material exists in a crystal lattice of high crystal symmetry then less reciprocal space needs to be covered in order to obtain a unique data set. Data collection on materials with high crystal symmetry can therefore be much shorter than on those with low crystal symmetry. Considering the crystal systems that are most common to organic and organometallic compounds, orthorhombic crystals would require a $90^{\circ}$ data collection in the equatorial plane in order to obtain a unique data set, whereas a hemisphere or full-sphere of data would be required for monoclinic or triclinic compounds, respectively.

Given the very complicated experimental conditions that are mandatory for observing structures of such transient species, it is incredibly difficult to obtain results with both high accuracy and good reciprocal space coverage, owing to the experimental time and X-ray intensity constraints imposed. However, it is precisely these experimental parameters that are paramount to obtaining data with high enough resolution for such an experiment to be successful in observing the subtle structural perturbations sought.

\section{Viability of charge-density studies within this development?}

Considering experimental parameters only, there are certain criteria that are deemed ideal for a charge-density study. (i) The crystal used should be of good quality so as to ensure maximum diffraction intensity and sharp peaks, and not to inflict unnecessary errors upon the resulting data due to variability in mosaicity, poor merging-equivalent or symmetryequivalent reflections, or contamination of Bragg peaks by

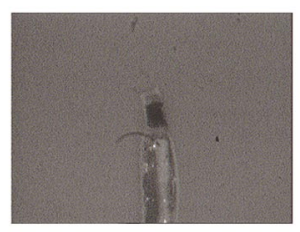

(a)

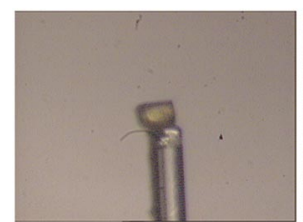

(c)

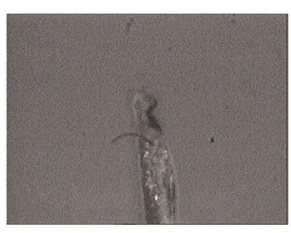

(b)

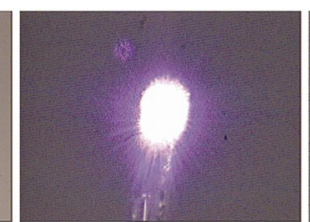

$(d)$

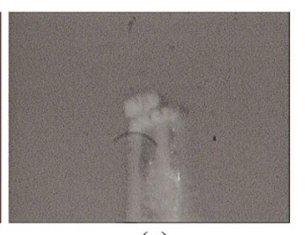

(e)
Figure 2

Crystals of $\left[\mathrm{HNCH}_{2} \mathrm{CH}_{2} \mathrm{NHCRe}\left(2,2^{\prime}\right.\right.$-bipyridine $\left.)(\mathrm{CO})_{3}\right] \mathrm{Br}$ mounted on a glass fibre, centred on the $\varphi$ axis of the ID9 beamline at the ESRF and subjected to a laser beam of $\lambda=400 \mathrm{~nm}$ at $50 \mu \mathrm{J}$ (Cole et al., 2003). (a) and (b) are different views of a crystal of $90 \mu \mathrm{m}$ diameter in the laser-beam direction post-irradiation; $(c),(d)$ and $(e)$ relate to the $40 \times 50 \times 60 \mu \mathrm{m}$ crystal ultimately used in the case study before, during and after laser irradiation, respectively. Note that the view of the crystal in $(e)$ is slightly obscured by ice that formed around the crystal during the experiment. Only amorphous contributions due to the icing were observed in the diffraction patterns. 
overlapping 'tails' from neighbouring peaks with very broad profiles. (ii) A compound with high crystal symmetry will result in greater data redundancy over a given area of reciprocal space and therefore provides intensities of greater accuracy as a result of better averaging. (iii) Molecules with small unit cells are most ideal since the data will give better statistics at high $\sin \theta / \lambda$ values than will data for molecules with large unit cells, for a given crystal size. If the experiment employs a one-dimensional detector, small unit cells are necessary in order to ensure that data can be collected to high $\sin \theta / \lambda$ resolution over a reasonably short period of time. (iv) Compounds that crystallize in centrosymmetric space groups are best since they are void of the uncertainties in phases that make the stability of charge-density analyses of non-centrosymmetric compounds very sensitive and sometimes problematic. (v) Low-temperature data collection ensures that thermal vibrations are reduced as far as possible, thereby minimizing the diminution of diffraction intensity due to thermal smearing effects. (vi) Slow data acquisition will result in the most statistically accurate collection of data frames and thence the best internal statistical agreement of data. (vii) The highest possible $\sin \theta / \lambda$ resolution of data should be acquired so that both the valence and the core electron density can be modelled adequately. (viii) Data redundancy should be as high as possible within the time feasibility of an experiment. Obviously, the higher the crystal symmetry of a compound, the more redundancy can be acquired over a given period of time. It is most preferable, however, to collect a full sphere of data at least out to a given resolution in a charge-density study of any compound, not just to ensure data redundancy but also to allow good absorption corrections and to check for systematic errors or data outliers, for example, due to beam-stop obstruction.

Considering these factors in relation to photoinduced experimental considerations, one can foresee many conflicts.

For studying long-lived or metastable states, the experimental side of a charge-density study is generally feasible as the experiment can be laboratory-based, it is free of laserinduced crystal decay once the light-induced state has been reached, and data collection on each component will take the same length of time as a conventional charge-density experiment. However, where structural changes are very large, as they are generally in photoisomerization studies, for example, where substantial atomic displacement takes place, subsequent data analysis is likely to be highly problematic. This is because the concomitance of the minor photoinduced state with the major ground-state component would make such a study akin to performing a charge-density study on a statically disordered compound. Charge-density theory, currently implemented in data analysis routines, would not be able to accommodate the minor photoinduced component as some of its electron density would overlap in space directly with electron density from part of the major (ground-state) component of the molecule. Any attempt to model electron density in the same volume of space by two sets of parameters is likely to fail since the sets of parameters would be too highly correlated statistically. Moreover, insurmountable space partitioning issues are likely to occur where electron-density contributions from two different atoms overlap in space.

Exceptionally, charge-density studies may be possible on one identified series of metastable light-induced compounds: iron-based spin-crossover complexes that undergo low-spin to high-spin electronic transitions, as evidenced by the expansion of the $\mathrm{Fe}-\mathrm{N}$ bond lengths by $\sim 0.2 \AA$ (Elhaik et al., 2003, and references therein; Kusz et al., 2000, 2001; Marchivie et al., 2002). The metastable state of these complexes is stabilized at low temperature; the elemental make-up of the materials is advantageous for charge-density studies, containing iron as the heaviest element; and, most importantly, the photoinduced structural change manifests itself by a subtle bond-length elongation that causes no overlap with other atoms and practically $100 \%$ photoconversion is possible without crystal fracture, thus avoiding the need to consider the light-induced structure in the fashion of static disorder. Indeed, singlecrystal polarized neutron diffraction studies have already been carried out to reveal topological maps of their magnetization densities (Goujon et al., 2003).

For studying transient photoinduced effects, the most notable problem relates to the time and accuracy issue described in the previous section. If basic structural determination of transient photoinduced states requires very long data-collection times that provide a coverage of reciprocal space for just a unique data set, where its accuracy is often compromised in order to afford a data collection over a reasonable time, surely charge-density studies are not viable without considerable improvements in experimental efficiency or marked changes in experimental strategies.

The prerequisite of synchrotron radiation for time-resolved experiments also tests the viability of charge-density studies. Whilst an increasing number of examples of charge-density studies from synchrotron sources have been reported over the past few years (see, for example, Dittrich et al., 2002; Flaig et al., 2002; Lippmann et al., 2003; Overgaard et al., 2002; Overgaard, Hibbs et al., 2003; Overgaard, Larsen et al., 2003; Pillet et al., 2003; Wagner et al., 2002; Whitten et al., 2004), there are still relatively few such studies conducted overall, leaving the field underdeveloped. It would therefore seem most appropriate for further developments in conventional chargedensity studies to be undertaken prior to branching out to charge-density studies on time-resolved X-ray diffraction data. Moreover, for materials where the photoinduced lifetime $\tau_{\mathrm{o}}<\mu \mathrm{s}$, time-resolved X-ray diffraction experiments are presently only possible on the ID9 beamline at the ESRF, Grenoble, France, and yet this beamline has only one degree of freedom for crystal motion (a $\varphi$ axis) and as such it is not suitable for charge-density experiments.

Any charge-density study on time-resolved photoinduced $\mathrm{X}$-ray diffraction data must also be able to account for thermal diffuse scattering (TDS) effects. This requirement arises because the photoinduced motion of atoms and/or changing electronic distributions in a molecule makes the presence of TDS implicitly likely.

There is also a resolution issue, and it is in fact very instructive to simulate a priori the structure factors that one 
would expect for such an experiment by calculating them from the reference ground-state crystal structure, which is altered artificially by successively increasing the relevant bond distance that is expected to be involved in the electronic perturbation. Thence, one can select out those reflections that are most altered by such a perturbation and plot these as a function of changing intensity due to the extent of change in bond distance. One can then consider the level of resolution that is required to observe a structural change using singlecrystal X-ray diffraction. Moreover, one can use the most sensitive reflections as controls during such an experiment. An example of such a simulation is given in Fig. 3.

In common with photoisomerization studies, the majority of compounds of scientific interest in transient light-induced physical processes are organometallic and frequently include second- and third-row transition metals. Potential chargedensity studies on these materials therefore suffer the same problems of inherent lack of theory as those for photoisomerization studies. Indeed, there is presently only one example of a time-resolved single-crystal $\mathrm{X}$-ray diffraction study and that pertains to a $\left[\mathrm{Pt}_{2}\left(\mathrm{H}_{2} \mathrm{P}_{2} \mathrm{O}_{5}\right)_{4}\right]^{4-}$ anion in which the $\mathrm{Pt}$...Pt distance is found to shorten in the excited state

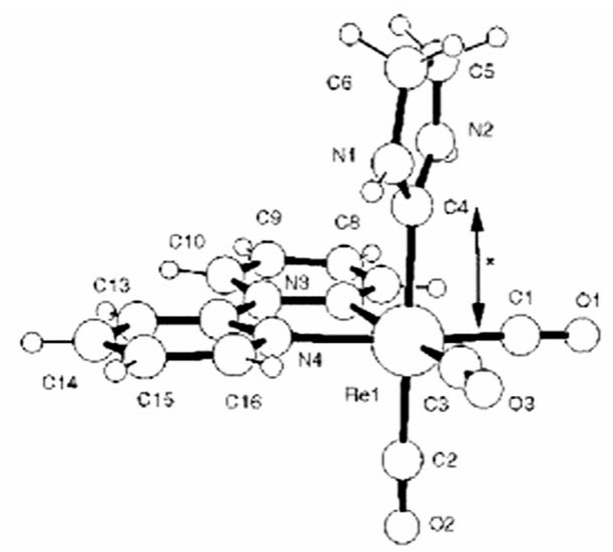

(a)

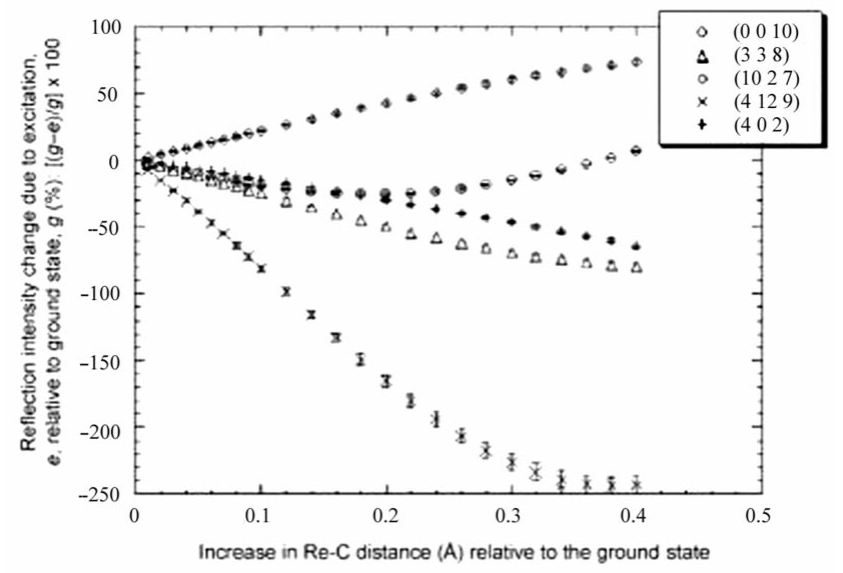

(b)

Figure 3

(a) The structure of the $\left[\mathrm{HNCH}_{2} \mathrm{CH}_{2} \mathrm{NHCRe}\left(2,2^{\prime} \text {-bipyridine }\right)(\mathrm{CO})_{3}\right]^{+}$ cation, showing the direction of the displacement of the diimine carbene ligand used to compute the simulated $F_{c}^{2}$ values, the relative intensity changes of the reflections that were found to most vary are plotted in $(b)$ (Cole et al., 2003).
(Kim et al., 2002). Bearing in mind the presence of platinum, this alone would render a charge-density study untenable using present methodologies.

From a materials-centred perspective, certain optical processes that are of great interest occur only in non-centrosymmetric compounds, for example nonlinear optical processes such as second-harmonic generation. This fact affords a further complication, since centrosymmetric molecules are best suited to charge-density studies, although such studies on non-centrosymmetric materials are possible if sufficient care is taken over their data analysis (see, for example, Cole et al., 2002; Smith et al., 1997; Spackman \& Byrom, 1997).

For time-resolved photoinduced studies where $\tau_{\mathrm{o}}$ is particularly short (ns-ps) and/or where the photoconversion percentage is very low, it is thought best to analyse difference diffraction intensity data rather than the absolute diffraction intensities (Coppens et al., 1998). This method is adopted because the differences will be freer from systematic errors and will be more statistically significant than the absolute intensities. The use of response ratios, the form in which these differences are presented (see equation below), for analysing small changes in structure has been demonstrated previously via the study of subtle piezoelectric structural perturbations (Paturle et al., 1991). This approach obviously has a fundamental bearing on any charge-density study, since analytical routines will need to be modified accordingly to allow for the analysis of such differences.

$$
\eta_{h k l}=\frac{I_{\mathrm{on}}(h k l)-I_{\mathrm{off}}(h k l)}{I_{\mathrm{off}}(h k l)}=\frac{I_{\mathrm{on}}(h k l)}{I_{\mathrm{off}}(h k l)}-1
$$

In photoinduced studies of any sort that involve organic or organometallic compounds, there is one final important consideration for judging the applicability of a compound for a charge-density study: that of the need for complementary hydrogen atomic coordinates and anisotropic displacement parameters from neutron diffraction, such that one can carry out a combined X-ray and neutron diffraction or similar type of analysis. As mentioned earlier, the majority of organic and organometallic compounds have an optical penetration depth of the order of micrometres. The normal crystal size requirements for neutron diffraction (minimum of $1 \mathrm{~mm}^{3}$ for most materials at a neutron source of reasonable neutron-beam flux) therefore render photoinduced studies untenable, except in the rare cases where the optical extinction coefficient of a material is exceptionally low. Sodium nitroprusside is one celebrated example of this (Rüdlinger et al., 1991, 1992). 'Insitu' charge-density studies on photoinduced materials are therefore likely to be confined to exploiting X-ray data only, if such studies are ever deemed viable.

\section{Need for further development and complementary tools}

It is apparent from the above considerations that the requirements for 'in-situ' charge-density studies on photoinduced structures are far from ideal and in fact are such that 
these studies are unlikely to be viable under present circumstances. However, at least some of the factors that currently preclude such charge-density studies in this regard may eventually be circumvented by future experimental and theoretical developments.

There is an increasing drive in performing charge-density studies at synchrotron sources, which has been aided by recent advances in detector technology. Whilst image plates still tend to provide a better dynamic range for data collection, chargecoupled-device (CCD) technology has been continually improving at a revolutionary rate over the past few years, in terms not only of dynamic range but also of greater overall sensitivity, sharper point-spread function, reduced detector readout time and improved data reproducibility. Detectors with substantially higher efficiency will be key to the viability of charge-density studies on transient species, since the severe data-collection time constraints are paramount to the present preclusion of such investigations.

Alternatively, the technology behind time-gated detectors, which is also currently developing at a rapid pace, may in fact hold the key to future potential feasibility of this research area, since the technology could circumvent the present need for pulsing X-rays entirely if applied suitably. In this scenario, a continuous X-ray source would be used in combination with a pulsed laser beam, which would be synchronized to the timegating detector electronics such that the X-ray detector timeseparates 'light on' and 'light off' signals as they arrive at the detector in an alternating fashion, but as a continuous beam. If this technology could be realized, it could eradicate the compromises that one is currently subjected to on account of $\mathrm{X}$-ray duty-cycle restrictions. Future developments in timegated detectors should therefore be observed with great interest given this potential for charge-density studies of timedependent processes.

If the future of time-resolved photoinduced charge-density studies remains in pulsed X-ray sources, however, their feasibility will be largely improved by the incorporation of time-resolved requirements into the designs of the new synchrotron sources and beamlines that are currently being planned and built around the world. The Advanced Photon Source (APS), Argonne, USA, and SPring-8, Japan, are particularly noteworthy as new synchrotron sources for timeresolved diffraction, since the synchrotron of the APS has an even shorter temporal structure (20 ps) than the ESRF, which is currently leading in time-resolved studies. Moreover, one of the focuses of the Chem-CARS beamline at the APS is dedicated to charge-density studies, whilst another focus is on time-resolved studies for which a mechanical chopper that realizes X-ray pulses (Ozawa et al., 1998) is already in place for studies down to $50 \mu \mathrm{s}$. The combination of these two goals impinges largely on the objectives of this paper. The APS is also one of the brightest X-ray sources in the world $(7 \mathrm{GeV})$, only SPring- 8 being brighter $(8 \mathrm{GeV})$. Such brightness may be used to overcome the major X-ray duty-cycle limitations that presently render transient structures so elusive.

The new wave of forthcoming medium-energy synchrotrons, notably Diamond and Soleil, in the UK and France, respectively, may also afford unique opportunities for photoinduced charge-density studies. At the Diamond facility, (laser)pump-(X-ray)probe time-resolved small-molecule crystallography and charge-density studies are two of the six key areas of structural science that have been targeted for the approved single-crystal diffraction beamline (Clegg et al., 2003). Moreover, the design of this beamline has incorporated specifications for anomalous X-ray scattering (AXS) studies, since this is one of the other targeted areas of structural science. AXS could be exploited very usefully in photoinduced studies in order to increase the contrast of the light-induced structure over that of the dominant ground state, in the area of expected change within a molecule. In luminescent organometallic materials, for example, the change anticipated frequently manifests itself in a change in $M-X$ bond distance. A diffraction experiment could be performed readily by collecting structural data first using X-rays of a wavelength close to that of the absorption edge of this metal and then using X-rays at a wavelength far from the edge. The difference between the two experiments would reveal a change in the atomic form factor explicitly due to the metal. The use of AXS in this fashion, proposed by Cole and co-workers (Cole et al., 2003), has yet to be exploited in practice but stands to be a very powerful tool in analysing the commonly subtle lightinduced structural changes in metal-containing compounds in the future. The beamline design at the Diamond synchrotron would be well suited in this regard. The Cristal beamline planned for the new Soleil synchrotron project is likely to provide similar experimental possibilities to the single-crystal diffraction beamline at Diamond and will include a diffractometer that is dedicated exclusively to charge-density experiments. These small-molecule crystallography beamlines are due to be online in 2006 and 2007 at Soleil and Diamond, respectively.

New technology that affords femtosecond X-ray pulses at a synchrotron, devised by a team at the Advanced Light Source, LBNL, California (Schoenlein et al., 1996), may also be incorporated into the designs of one of the synchrotrons presently being constructed. Here, femtosecond X-ray pulses are created by 'tickling' the electron beam in the linear accelerator of a synchrotron with a femtosecond-pulsed laser in such a way that a fraction of the photons and electrons interact, resulting in photons that are scattered with regular femtosecond-pulsed X-ray energies. Currently, proof of principle has been demonstrated but the resulting X-ray flux has been considered to be too weak for viable application. However, if improvements overcome this barrier, the exploitation of this technique stands to open up a whole new time regime ( $\mathrm{ps}-\mathrm{fs}$ ) of photoinduced X-ray diffraction studies that is presently inaccessible. The fact that chemical bond making and bond breaking typically occurs on the femtosecond timescale makes such a possibility particularly exciting since one could envisage, for example, molecular movies of chemical reactions, thus revealing three-dimensional images of their mechanisms through a time function of structural manifestations. The proposition of charge-density studies of such dynamic and predominantly irreversible evolutionary 
processes may seem outlandish presently, especially given the substantial difficulties described above for just obtaining transient structures that are captured by repetitive pumpprobe technology, but the unpredictability of future technological advances may yet pave the way to the seemingly impossible.

Thus far, considerations have been based on monochromatic oscillatory diffraction techniques. Sometime in the future, one may be able to consider employing Laue rather than monochromatic oscillatory-based diffraction techniques for charge-density studies. Laue diffraction has an enormous intrinsic advantage in terms of the data-collection time required for collecting sufficient data for determining a structure, being orders of magnitude faster than an analogous monochromatic oscillatory-based structure determination. Hitherto, Laue-based diffraction methods have been held back by poor atomic resolution (owing to inefficient deconvolution of overlapping reflections of different wavelengths) and inadequate, and sometimes patchy, reciprocal space coverage. Current developments in this area are, however, evidencing a 'coming-of-age' of Laue diffraction, the impetus on these advances stemming from the biological sphere of diffraction (Ren et al., 1999). If the current concerns about Laue diffraction with respect to charge-density studies can ultimately be overcome, it is possible that Laue diffraction may become the salvation of charge-density studies of transient photoinduced species. The ID9 beamline at the ESRF, Grenoble, France, already houses the experimental setup for Laue-based photoinduced diffraction and this setup has demonstrated enormous success in realizing time-resolved photoinduced structural conformational changes in important biological species (Genick et al., 1997; Perman et al., 1998; Srajer et al., 1996), albeit with non-atomic resolution.

One thing that is certain is the rapidly growing success of complementary density functional theory (DFT) for chargedensity studies. At such a time when charge-density studies on photoinduced species do become viable, it will be very important to assess the validity of the early experiments in this regard via complementary DFT calculations, as far as such an assessment is possible.

The exploitation of complementarity with regards to other experimental techniques is also important for realizing the feasibility of photoinduced charge-density studies. In particular, the current developments in time-resolved EXAFS and IR studies are very pertinent here since, whilst they cannot contribute directly to any charge-density study, they are able to provide important a priori information as to the structural nature and extent of the photoinduced change sought in a given compound. The comprehensive reviews by Saes et al. (2004, and references therein) and George \& Turner (1998) provide further details of time-resolved EXAFS and IR developments, respectively.

\section{Concluding remarks}

For the time being, it therefore seems that 'in-situ' chargedensity studies on photoinduced species are not viable, perhaps with the exception of the study of metastable photoinduced species such as iron-containing spin-crossover transitions where there are only very subtle structural changes, in this case, bond-length changes of the order of $\sim 0.2 \AA$. There are a plethora of experimental and theoretical constrictions to overcome in order to make such charge-density studies viable in all other cases, and whilst there are various encouraging developments underway to alleviate some of these problems, progress will inevitably take some time. In the meantime, however, one may still be able to carry out structural studies that are more extensive than a conventional IAM refinement. For example, a kappa-refinement may be possible since this would not require an inordinate amount of data or refined parameters to be defined. Thus, one can begin to consider bonding effects in a limited fashion, but we look forward to a time someway ahead in the future when much fuller bonding descriptions may be finally realized.

The author thanks the Royal Society for a University Research Fellowship, St Catharine's College, Cambridge, for a Senior Research Fellowship and the European Science Foundation for funding that enabled her to participate in the PESC exploratory workshop in charge-density analysis, Sandberg, Denmark (June 2003), her contribution from which forms the basis of this paper.

\section{References}

Carducci, M. D., Pressprich, M. R. \& Coppens, P. (1997). J. Am. Chem. Soc. 119, 2669-2678.

Clegg, W., Cole, J. M., Morris, R., Raithby, P. R., Teat, S. J., Wilson, C. C., Wilson, C., Evans, J. \& Smith, M. (2003). Diamond Light Source Technical Report, Sci-BLP-028-0101 (for ease of availability, see also: http://www.diamond.ac.uk/Publications/1987/Sciblp-028-0101.pdf).

Cole, J. M., Goeta, A. E., Howard, J. A. K. \& McIntyre, G. J. (2002). Acta Cryst. B58, 690-700.

Cole, J. M., Raithby, P. R., Wulff, M., Schötte, F., Plech, A., Teat, S. J. \& Bushnell-Wye, G. (2003). Faraday Discuss. 122, 119-129.

Coppens, P., Fomitchev, D. V., Carducci, M. D. \& Culp, K. (1998). J. Chem. Soc. Dalton Trans. pp. 865-872.

Dittrich, B., Koritsanszky, T., Grosche, M., Scherer, W., Flaig, R., Wagner, A., Krane, H. G., Kessler, H., Riemer, C., Schreurs, A. M. M. \& Luger, P. (2002). Acta Cryst. B58, 721-727.

Elhaïk, J., Money, V. A., Barrett, S. A., Kilner, C. A., Radosavljevic Evans, I. \& Halcrow, M. A. (2003). J. Chem. Soc. Dalton Trans. pp. 2053-2060.

Flaig, R., Koritsanszky, T., Dittrich, B., Wagner, A. \& Luger, P. (2002). J. Am. Chem. Soc. 124, 3407-3417.

Genick, U. K., Borgstahl, G. E. O., Ng, K., Ren, Z., Pradervand, C., Burke, P. M., Srajer, V., Teng, T.-Y., Schildkamp, W., McRee, D. E., Moffat, K. \& Getzoff, E. D. (1997). Science, 275, 1471-1475.

George, M. W. \& Turner, J. J. (1998). Coord. Chem. Rev. 177, 201.

Goujon, A., Gillon, B., Gukasov, A., Jeftic, J., Nau, Q., Codjovi, E. \& Varret, F. (2003). Phys. Rev. B, 67, 220401-4.

Kim, C. D., Pillet, S., Wu, G., Fullagar, W. K. \& Coppens, P. (2002). Acta Cryst. A58, 133-137.

Kovalevsky, A. Y., Bagley, K. A., Cole, J. M. \& Coppens, P. (2003). Inorg. Chem. 42, 140-147.

Kovalevsky, A. Y., Bagley, K. A. \& Coppens, P. (2002). J. Am. Chem. Soc. 124, 9241-9248.

Kusz, J., Spiering, H. \& Gütlich, P. (2000). J. Appl. Cryst. 33, 201-205. Kusz, J., Spiering, H. \& Gütlich, P. (2001). J. Appl. Cryst. 34, 229-238. 
Lippmann, T., Blaha, P., Andersen, N. H., Poulsen, H. F., Wolf, T., Schneider, J. R. \& Schwarz, K. H. (2003). Acta Cryst. A59, 437-451.

Marchivie, M., Guionneau, P., Howard, J. A. K., Chastanet, G., Letard, J.-F., Goeta, A. E. \& Chasseau, D. (2002). J. Am. Chem. Soc. 124, 194-195.

Overgaard, J., Hibbs, D. E., Rentschler, E., Timco, G. A. \& Larsen, F. K. (2003). Inorg. Chem. 42, 7593-7601.

Overgaard, J., Iversen, B. B., Palii, S. P., Timco, G. A., Gerbeleu, N. V. \& Larsen, F. K. (2002). Chem. Eur. J. 8, 2775-2786.

Overgaard, J., Larsen, F. K., Schiott, B. \& Iversen, B. B. (2003). J. Am. Chem. Soc. 125, 11088-11099.

Ozawa, Y., Pressprich, M. R. \& Coppens, P. (1998). J. Appl. Cryst. 31, 128-135.

Paturle, A., Graafsma, H., Sheu, H.-S. \& Coppens, P. (1991). Phys. Rev. B, 43, 14683-14691.

Perman, B., Srajer, V., Ren, Z., Teng, T.-Y., Pradervand, C., Ursby, T., Bourgeois, D., Schotte, F., Wulff, M., Kort, R., Hellingwerf, K. \& Moffat, K. (1998). Science, 279, 1946-1950.

Pillet, S., Wu, G., Kulsomphob, V., Harvey, B. G., Ernst, R. D. \& Coppens, P. (2003). J. Am. Chem. Soc. 125, 1937-1949.

Ren, Z., Bourgeois, D., Helliwell, J. R., Moffat, K., Srajer, V. \& Stoddard, B. L. (1999). J. Synchrotron Rad. 6, 891-917.

Rüdlinger, M., Schefer, J., Chevrier, G., Furer, N., Güdel, H. U., Haussühl, S., Heger, G., Schweiss, P., Vogt, T., Woike, T. \& Zöllner, H. (1991). Z. Phys. B Condens. Matter, 83, 125-130.
Rüdlinger, M., Schefer, J., Vogt, T., Woike, T., Haussühl, S. \& Zöllner, H. (1992). Physica B, 180-181, 293-298.

Saes, M., Bressler, C., van Mourik, F., Gawelda, W., Kaiser, M., Chergui, M., Bressler, C., Grolimund, D., Abela, R., Glover, T. E., Heimann, P. A., Schoenlein, R. W., Johnson, S. L., Lindenberg, A. M. \& Falcone, R. W. (2004). Rev. Sci. Instrum. 75, 24-30.

Schoenlein, R. W., Leemans, W. P., Chin, A. H., Volfbeyn, P., Glover, T. E., Balling, P., Zolotorev, M., Kim, K. J., Chattopadhyay, S. \& Shank, C. V. (1996). Science, 274, 236-238.

Smith, G. T., Mallinson, P. R., Frampton, C. S., Farrugia, L. J., Peacock, R. D. \& Howard, J. A. K. (1997). J. Am. Chem. Soc. 119, 5028-5034.

Spackman, M. A. \& Byrom, P. G. (1997). Acta Cryst. B53, $553-564$.

Srajer, V., Teng, T.-Y., Ursby, T., Pradervand, C., Ren, Z., Adachi, S.-I., Schildkamp, W., Bourgeois, D., Wulff, M. \& Moffat, K. (1996). Science, 274, 1726-1729.

Wagner, A., Flaig, R., Zobel, D., Dittrich, B., Bombicz, P., Strumpel, M., Luger, P., Koritsanszky, T. \& Krane, H. G. (2002). J. Phys. Chem. A, 106, 6581-6590.

Whitten A. E., Dittrich, B., Spackman, M. A., Turner, P. \& Brown, T. C. (2004). J. Chem. Soc. Dalton Trans. pp. 23-29.

Wulff, M., Schotte, F., Naylor, G., Bourgeois, D., Moffat, K. \& Mourou, G. (1997). Nucl. Instrum. Methods Phys. Res. Sect. A, 398, 69-84. 